\title{
Turbulent jet stability increased by ribs inside the nozzle - Stereo PIV measurement one diameter past the nozzle
}

\author{
Daniel Duda ${ }^{1 *}$, Vladimír Abrhám ${ }^{1}$, Václav Uruba ${ }^{1,2}$, and Vitalii Yanovych ${ }^{1}$ \\ ${ }^{1}$ University of West Bohemia in Pilsen, Univerzitní 22, Pilsen, Czech Republic \\ ${ }^{2}$ Intstitute of Thermomechanics of the Czech Academy of Sciences, Dolejškova 5, Prague, Czech \\ Republic
}

\begin{abstract}
We observe that decreasing the inner nozzle surface by adding longitudinal ribs increases the jet stability in terms of the amount of turbulent kinetic energy in the near shear layer. We try to explain our observation as a stabilization effect of secondary flow vortices emerging in the corners of the ribs. These stream-wise vortices damage the development of larger-scale structures in the near shear layer. This explanation is supported by autocorrelation function of the stream-wise velocity component, which displays slightly smaller integral length-scale in the case with ribs than in the case of smooth nozzle. The experiment is performed at Reynolds number $2.2 \times 10^{5}$ (based on the nozzle diameter $50 \mathrm{~mm}$ ); the Stereo-PIV (Particle Image Velocimetry) measurement takes place at the plane perpendicular to the jet axis one diameter past the nozzle exit. Optical 3D scanner controls the real nozzle geometry. This article presents preliminary measurement at single position and single velocity only; further exploration of this problem is needed.
\end{abstract}

\section{Introduction}

Jet is the flow structure created by fluid leaving some nozzle or orifice into surroundings with different velocity. In the near stage, we distinguish the jet core surrounded by the growing shear layer, in later stages, the jet core vanishes, the stream-wise velocity profile converges to Gaussian growing in space and weakening in velocity $[1,2]$. The character of turbulence converges to self-similar isotropic and homogenous (at least locally) turbulence of Kolmogorovy style until the dissipative length-scale does not overgrow the integral lengthscale. Back to the beginning: the shear layer near the nozzle is often though to display the clearest natural living environment for Kelvin-Helmholtz instability, which is one of the basic flow instabilities, because it takes places in all shears and transverse velocity gradients everywhere within the flowing fluids. It occurs even inside the vortices [3] leading to the fractal nature of turbulence [4].

Jet flows have many engineering applications from chemical mixing (we want high turbulence without strong winds) up to propulsion (we want maximum velocity without

* Corresponding author: dudad@kke.zcu.cz 
turbulent loses). Therefore, it would be nice to control the jet stability by manipulating the last solid part in the problem - the nozzle.

\section{Experimental setup}

The air jet is powered via the radial compressor with inlet from the atmosphere. The outlet flow is accelerated by using a smooth convergent nozzle from diameter $200 \mathrm{~mm}$ to $50 \mathrm{~mm}$. The airflow is further modified by using the studied nozzle with or without ribs on its inner surface (see Fig. 1 and 2 for easier imagination). Diameter of this second nozzle is constant $D=50 \mathrm{~mm}$ and its length is $100 \mathrm{~mm}$, i.e. $2 D$. This nozzle has been created in three variants: (a) with a smooth inner cylindrical surface, (b) with 6 longitudinal ribs and (c) with 12 longitudinal ribs with half thickness than in the (b) case in order to keep the blockage ratio constant. The height of the ribs linearly increases from 0 at the nozzle inlet to $2.5 \mathrm{~mm}$ (i.e. 5 $\%$ of $D$ ) at the nozzle outlet. The blockage ratio is $1.5 \%$ in both variants with ribs; this has been compensated during the measurement by increasing the compressor power by this percentage, when measuring the flow past the smooth nozzle in order to keep the core velocity comparable. All the nozzles have been created by using 3D printer [6] Raise 3D from polymerized lactic acid PLA [5].

The accuracy of 3D printing procedure has been checked by using the optical 3D scanning system GOM Atos Core 300. This measuring is based on the projecting fringes of varying width and observing their displacement $[7,8,9]$. Results in Fig. 1 show reasonable accuracy with deviations up to $0.3 \mathrm{~mm}$ from the planed geometry. One of the limitation of the $3 \mathrm{D}$ scanning technology is the problematic scanning of details inside voids [8]; therefore, we are not able to check the quality of the ribs in the middle of the nozzle length. However, at the outlet, they are scanned well. The systematic error of printing lies in the sharpness of the edges, which display radius around half millimeter (see Fig. 1 right), although they was planned to be sharp. The nozzle diameter was smaller by $0.08 \mathrm{~mm}$ (i.e. $0.16 \%$ ) than the planned one. The rib thickness is larger by $7 \%$. On the other hand, the circularity of cylindrical surfaces is very good (see Fig. 1 left panel).

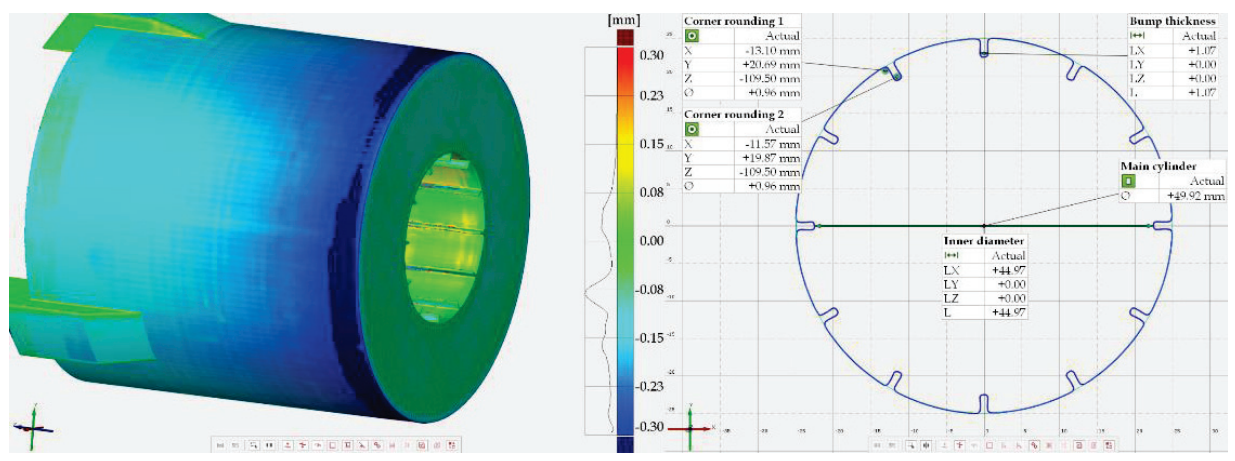

Fig. 1. The result of optical 3D scanning of the nozzle modification (c) with 12 ribs. Left panel shows the deviation from ideal geometry represented by the CAD (computer-aided design) model used for the $3 \mathrm{D}$ printing. Right panel shows the section at the exit $(0.5 \mathrm{~mm}$ from it in order to avoid noise) with denoted actual dimensions. In a case of ideal 3D printer, the corner roundings should have diameter 0 , the rib (bump) thickness might be exactly $1 \mathrm{~mm}$, the main cylinder diameter $\mathrm{D}$ should be $50.0 \mathrm{~mm}$ and inner diameter might be $45.0 \mathrm{~mm}(\mathrm{D}-2 \times$ rib height $)$.

The flow in the jet produced by the described nozzles is measured by using Stereo PIV method [10]. In the fluid, there are small droplets of diameter around $1 \mu \mathrm{m}$, thus the inertial (volume) forces are much smaller than the viscous (surface) forces affecting trajectories of 
such a small particles and therefore their movement can be interpreted as the movement of the fluid around them [10]. Double-pulse solid-state laser defocused via cylindrical lenses into a sheet illuminates these particles. The illuminated area is normal to the jet direction; it lies $50 \mathrm{~mm}$, i.e. $1 D$ past the nozzle outlet. Particles in this area are observed by pair of cameras from two directions rotated by $45^{\circ}$ against the jet direction. The position of observed plane is displayed in Fig.1 top. We use commercial the Stereo PIV system from the company Dantec, whose software Dantec Dynamic Studio is used for operating the equipment and for processing the data in terms of converting the pairs of double-snapshots gray-scale photographs into single 3D velocity vector fields; the details can be found elsewhere [10,11]. The air velocity is $62.7 \mathrm{~m} / \mathrm{s}$ corresponding to diameter-based Reynolds number $2.22 \cdot 10^{5}$.

\section{Results}
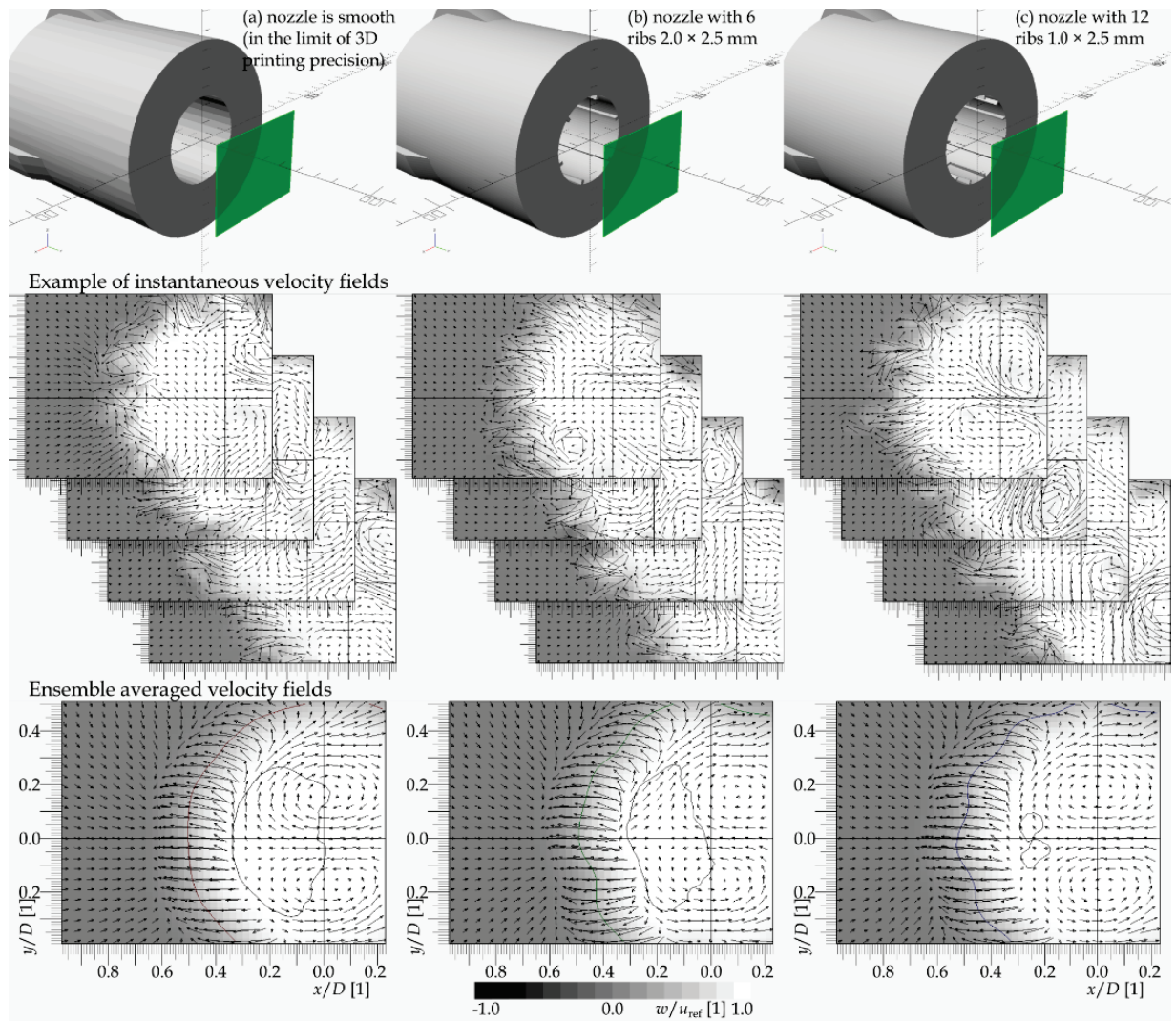

Fig. 2. (Top) The relative position of the measured area to the modified nozzle. (Middle) example of few instantaneous velocity fields. (Bottom) Ensemble average of 628 snapshots. The in-plane velocity components are displayed as arrows (only every second vector is displayed). Stream-wise component (normal to the measured plane) is represented by the gray-scale.

The studied area covers partly the jet core, the shear layer and the surroundings as well. The ensemble average velocity fields for the three nozzles are displayed in Fig. 2 bottom. We see the jet core area with "table-top" in the right-hand side of the figures, in the middle, there is the bow of the shear layer, where the stream-wise velocity component perpendicular to the measured plane (denoted $w$ in this paper) decreases. Due to the mass conservation, the 
direction of the flow deflects away from the jet axis, which is observable as increase in the magnitude of in-plane velocity components (denoted $u$ and $v$ ). The ribs in the nozzle slightly affect the shape of the average velocity creating waves on the isotach at $1 / 2 u_{\text {ref. }}$. We see an imperfectness in the average velocity - a pair of stream-wise vortices in the jet core. The used wind tunnel is not fully completed yet, it has not the grids in the settlings chamber. We hope that for the future experiments, the chamber will be completed and these large-scale vortices will no longer perturb our measurements.

The shear layer is home of the turbulence created firstly via the Kelvin-Helmholtz instability and later via reconnections of larger-scale vortices, vortex stretching and other mechanisms typical for turbulent flows. The energy source and entropy bath for this show is the velocity gradient between the jet core and the ambient. The turbulent motion appears chaotic for a first look [4], and its amount can be expressed in terms of the turbulent kinetic energy TKE

$$
\mathrm{TKE}=\frac{1}{2}\left\langle u^{\prime 2}+v^{\prime 2}+w^{\prime 2}\right\rangle
$$

where $\langle\cdots\rangle$ means ensemble averaging, $u, v$ and $w$ are the fluctuating velocity components

$$
u^{\prime}=u-\langle u\rangle
$$

and other components analogically. This operation is known as Reynolds decomposition [12].

The spatial distribution of TKE is in Fig. 3 and Fig. 4 displays its development along the horizontal axis depicted in the spatial maps. Here we finally come to the effect mentioned in the title - roughening the nozzle surface leads to decrease in turbulent kinetic energy in the near shear layer. Therefore one can estimate the decrease in the turbulent transport of momentum and thus to better stability of the jet in later stages. (Although this is only assumption, we did not measure it yet.)
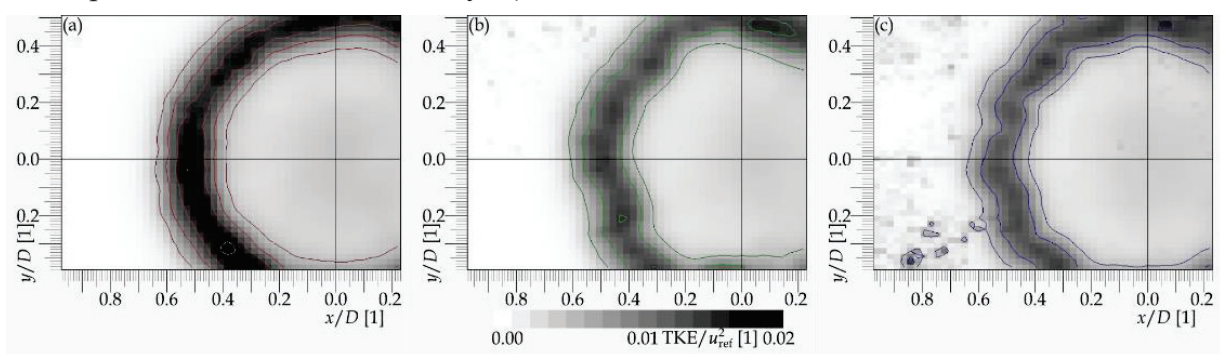

Fig. 3. Spatial distribution of turbulent kinetic energy TKE past three nozzle variants.
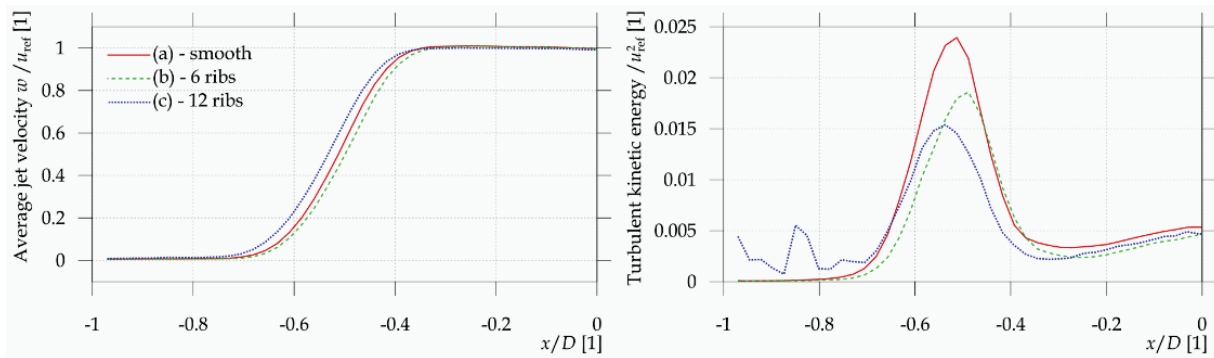

Fig. 4. Radial profile of the ensemble-average stream-wise velocity w (left) and Turbulent kinetic energy (right) along the $\mathrm{x}$-axis depicted in other figures. 


\section{Discussion}

We venture to explain this phenomenon by the presence of secondary flows [13] along the ribs $[14,15]$. This secondary flow takes the form of longitudinal vortices $[16,17,18]$, which, although are weak and disturbed by the surrounding turbulence, can prevent or, at least, delay the development of large-scale toroidal vortices of Kelvin-Helmholtz instability. The presence of stream-wise vortices past the ribs may appear in the averaged vorticity field even in higher distances from the nozzle, see Fig. 5. However, the in-plane fluctuations of the entire jet blur them in the ensemble-averaged data disturbed by experimental noise.
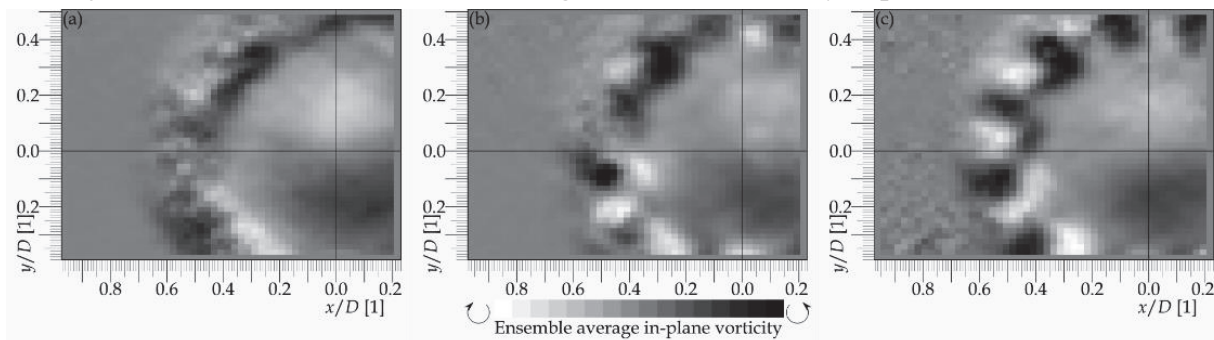

Fig. 5. The ensemble average of in-plane vorticity component (other vorticity components are not calculable as we do not know the gradients in stream-wise direction).

The vorticity $\omega$ displayed in gray-scale in Fig. 5 is calculated as the rotation of twodimensional velocity field

$$
\omega=\nabla \times\left(\begin{array}{l}
u \\
v
\end{array}\right)
$$

which is calculated on grid with spatial resolution $\Delta x$ and $\Delta y$ by using simple symmetrical differential scheme [18]

$$
\omega(x, y)=\frac{u(x, y+\Delta y)-u(x, y-\Delta y)}{2 \Delta y}-\frac{v(x+\Delta x, y)-v(x-\Delta x, y)}{2 \Delta x}
$$

Thus, it is only the $z$-component of vorticity as we do not know the velocity gradients in $z$ direction.

Although the instantaneous velocity fields display turbulent behaviour at the shear layer, the presence of regular weak stream-wise vortices leads to smaller length-scale of turbulence there [2]. The autocorrelation function $R_{w w}$ is widely used in flow research [19]. It represents somehow the "memory" of the system, i.e. a statistical connection of events separated by distance $\Delta r$

$$
R_{w w}(\vec{x}, \Delta \vec{r})=\frac{1}{\sigma_{w}(\vec{x})} \cdot \frac{1}{\sigma_{w}(\vec{x}+\Delta \vec{r})}\left\langle w^{\prime}(\vec{x}) \cdot w^{\prime}(\vec{x}+\Delta \vec{r})\right\rangle
$$

Fig. 6 shows the correlation function of stream-wise velocity component $w$. The localization of reference point $x$ has been chosen in four different locations: (1) in the surroundings near the shear layer; (2) in the center of shear layer; (3) at the beginning of shear layer (from the core) and (4) in the jet core axis. The case (2) is shown in Fig. 6 for all possible $\Delta r$ displaying the shape of correlation spot as well. Fig. 7 displays the comparison of the correlation function $R_{w w}$ along the vertical lines depicted in both figures. 

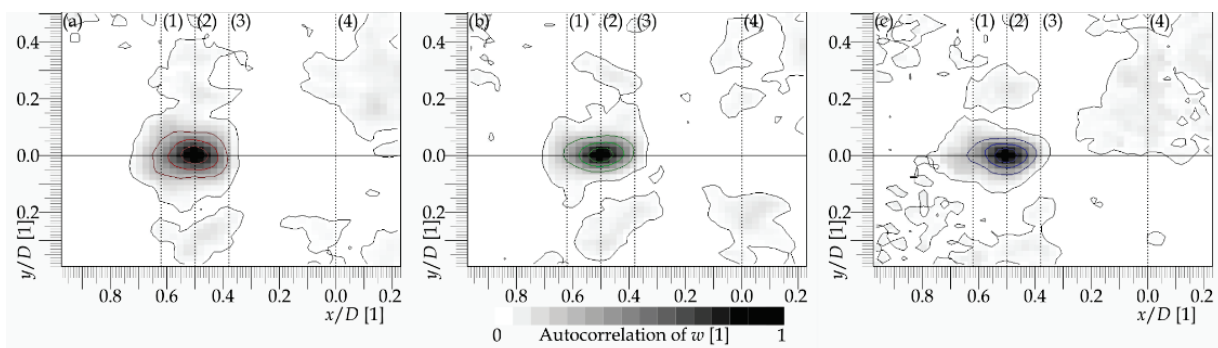

Fig. 6. The spatial correlation function of stream-wise fluctuation velocity $\mathrm{R}_{\mathrm{ww}}$. The cross-section points of lines (1) - (4) with the horizontal $x$-axis are the reference points of $R_{w w}$, the case (2) applies for this figure. The other cases are displayed in Fig. 7 only along the lines denoted (1) - (4).
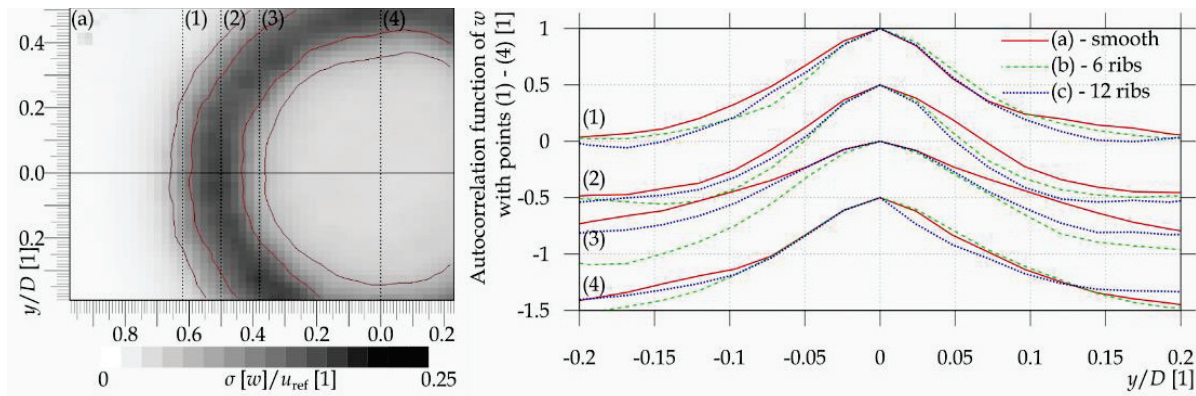

Fig. 7. (Right) the correlation function $\mathrm{R}_{\mathrm{ww}}$ along the lines (1) - (4) depicted in the left-hand side in the spatial distribution of standard deviation of $\mathrm{w}$.

The autocorrelation function $R_{w w}$ clearly displays that the fluctuations in the smooth case (a) are more spacious. This effect is stronger inside the shear layer, while in the jet core (4) and ambient (1) their spatial size is comparable.

\section{Conclusion}

We have observed that adding the ribs into the nozzle decreases the turbulent kinetic energy in the shear layer in the distance of 1 diameter past the nozzle exit. We suggest explanation, that the secondary corner vortices formed along the ribs may stabilize the shear layer against the large-scale vortices formed via the Kelvin-Helmholtz instability. Therefore, the fluctuations are slightly smaller in spatial terms.

There remain many questions to answer in the future: e.g., what happens later? Will the stabilization effect continue or not? In addition, what happens, when we twist the ribs? The swirling jets generally opens faster due to centrifugal forces [20,21].

The scanning was supported from ERDF under project Research Cooperation for Higher Efficiency and Reliability of Blade Machines (LoStr) No. CZ.02.1.01/0.0/0.0/16_026/0008389.

We thank in advance to the Grant Agency of the Czech Republic for supporting our project proposal, which will allow us to explore these fascinating phenomena deeper.

\section{References}

1. E. J. List, Turbulent jets and plumes, Annual Review of Fluid Mechanics 14, 189-212 (1982), doi: 10.1146/annurev.fl.14.010182.001201 
2. D. Duda, V. Uruba, Spatial spectrum from Particle Image Velocimetry data, Journal of Nuclear Engineering and Radiation Science, 5, 031901 (2019), doi: 10.1115/1.4043319

3. E. Amromin, Analysis of vortex core in steady turbulent flow, Physics of Fluids 19, 118108 (2007); doi: 10.1063/1.2813045

4. J. M. Redondo, A. K. Platonov, Self-similar distribution of oil spills in European coastal waters, Environmental Research Letters, 4, 014008 (2009), doi: 10.1088/17489326/4/1/014008

5. Inkinen, S., Hakkarainen, M., Albertsson, A.., Södergøard, A. From lactic acid to poly(lactic acid) (pla): Characterization and analysis of pla and its precursors. Biomacromolecules 12, 523-532 (2011) doi:10.1021/bm101302t.

6. Alsoufi, M.S., Elsayed, A.E., 2018. Surface roughness quality and dimensional accuracy - a comprehensive analysis of 100per-sonal/desktop cost-effective FDM 3D printer. Materials Sciences and Applications 09, 11-40. doi:10.4236/msa.2018.91002.

7. Zhang, C., Zhao, H., Gu, F., Ma, Y., 2015. Phase unwrapping algorithm based on multifrequency fringe projection and fringe background for fringe projection profilometry. Measurement Science and Technology 26. doi:10.1088/0957-0233/26/4/045203.

8. Mendricky, R., 2016. Determination of measurement accuracy of optical 3d scanners. MM Science Journal 2016, 1565-1572. doi:10.17973/MMSJ.2016_12_2016183.

9. Vagovský, J., Buranský, I., Görög, A., 2015. Evaluation of measuring capability of the optical 3d scanner, in: Procedia Engineering, pp. 1198-1206. doi:10.1016/j.proeng.2015.01.484.

10. Tropea, C., Yarin, A., Foss, J.F., 2007. Springer Handbook of Experimental Fluid Mechanics. Springer, Heidelberg, DE.

11. V. Kopecký, Laserová anemometrie v mechanice tekutin (2008)

12. D. Duda, V. Uruba, PIV of air flow over a step and discussion of fluctuation decompositions, AIP Conference Proceedings, 2000, 1, 020005 (2018) doi: $10.1063 / 1.5049912$

13. J. Nikuradse, Untersuchungen über die Geschwindigkeitsverteilung in Turbulenten Strijmungen (Ph.D. thesis), Univesty of Götingen, Götingen, 1926.

14. Z. Wang, N. Cheng, Time-mean structure of secondary flows in open channel with longitudinal bedforms, Adv. Water Resour. 29 (11) (2006) 1634-1649, doi: 10.1016/j.advwatres.2005.12.002.

15. M. Uhlmann, A. Pinelli, G. Kawara and A. Sekimoto, Marginally turbulent flow in a square duct, J. Fluid Mech. 588: 153-162 (2007) doi: 10.1017/S0022112007007604

16. D. Duda, J. Béma, V. Yanovych, P. Pavlíček, V. Uruba, Secondary flow of second kind in a short channel observed by PIV, European Journal of Mechanics / B Fluids 79, 444453 (2020), doi: 10.1016/j.euromechflu.2019.10.005

17. D. Duda, M. La Mantia and L. Skrbek, Streaming flow due to a quartz tuning fork oscillating in normal and superfluid He 4, Physical Review B 96 (2) 024519 (2017) doi: 10.1103/PhysRevB.96.024519

18. J. Bém, D. Duda, J. Kovařík, V. Yanovych, V. Uruba, Visualization of Secondary Flow in a Corner of a Channel, AIP Conference Proceedings 2189, 020003 (2019), doi: $10.1063 / 1.5138615$

19. E. O. Schulz-DuBois and I. Rehberg, Structure function in lieu of correlation function, Applied Physics 24: 323-329 (1981)

20. C. E. Cala, E. C. Fernandes, M. V. Heitor, S. I. Shtork, Coherent structures in unsteady swirling jet flow, Experiments in Fluids volume 40, 267-276 (2006), doi: 10.1007/s00348-005-0066-9

21. D. Duda, The PIV study of air flow past the counter-swirler 53983, MATEC Web of Conferences 168, 05004 (2018), doi: 10.1051/matecconf/201816805004 